Instituto Internacional de Investigación y Desarrollo Tecnológico Educativo INDTEC, C.A.

DOI: https://doi.org/10.29394/scientific.issn.2542-2987.2016.1.2.10.162-180

OAI-PMH: http://www.indteca.com/ojs/index.php/Revista Scientific/oai

\title{
La Educación Intercultural
}

\author{
Autora: María del Carmen Pérez Paredes \\ Universidad Nacional Experimental "Rafael María Baralt", UNERMB \\ miduam@hotmail.com \\ Mérida, Venezuela
}

\section{Resumen}

La educación intercultural se define y se concibe como un proceso continuo de desarrollo aprendizaje en todos los niveles educativos y en los ejes integradores de la Educación, donde el Estado con la participación de la familia y la sociedad promoverá la diversidad cultural en el proceso de educación ciudadana. Es por ello que el objetivo de este estudio consistió en analizar los aspectos de la educación intercultural con la finalidad de propiciar las potencialidades que garanticen su integración en las propuestas emanadas en los diseños curriculares. Se sustentó en la teoría de la complejidad (Morín, 2008, 2001a, 2001b) y la Teoría de la Educación Intercultural (Yampara, 2001). Metodológicamente fue una investigación analítica y documental por lo que se encuentra inserta en los estudios de desarrollo teórico. En ese sentido, se apoyó en autores como Manual de la Universidad Pedagógica Experimental Libertador (2003), Arias (2006:27), Sánchez (2000). Como resultado se obtuvo que los aspectos de la complejidad están presentes en la educación intercultural bajo la forma de principios tales como el principio dialógico, el principio recursivo, el principio hologramático, el principio de cultura, el principio de autopoiesis, el principio de identidad y, el valor del conocimiento y de saberes, concluyendo que es posible afirmar que la educación intercultural está presente en los principios de la complejidad y se encuentran representados en ella.

Palabras clave: educación intercultural; complejidad; principio de cultura; el principio de autopoiesis; diseño curricular. 


\title{
The Intercultural Education
}

\begin{abstract}
Intercultural education is defined and conceived as a continuous process of development learning at all educational levels and integrators axes of Education, where the state with the participation of the family and society promote cultural diversity in the educational process citizen. That is why the aim of this study was to analyze aspects of intercultural education in order to foster the potential to ensure their integration into the proposals coming in curriculum design. It was based on the theory of complexity (Morin, 2008, 2001a, 2001b) and the Theory of the Intercultural Education (Yampara, 2001). Methodologically was an analytical and documentary research which is inserted in studies of theoretical development. In that regard, it relied on authors such as Manual Pedagogical University Experimental Libertador (2003), Arias (2006: 27), Sánchez (2000). As a result it was found that aspects of complexity are present in the intercultural education in the form of principles such as the dialogic principle, the recursive principle, hologram tic principle, the principle of culture, the principle of autopoiesis, the principle of identity and the value of knowledge and wisdom, concluding that it can be said that intercultural education is present in the principles of complexity and are represented.
\end{abstract}

Keywords: intercultural education; complexity; culture principle; the principle of autopoiesis; curriculum design. 
No nos damos cuenta de la prodigiosa diversidad de los juegos de lenguaje cotidianos porque el revestimiento exterior de nuestro lenguaje hace que parezca todo igual.

Ludwig Wittgenstein

\section{Introducción}

La educación intercultural se define tácitamente como un derecho humano y un deber social para el desarrollo de la persona dentro de las pautas de diversidad y participación, con la finalidad de garantizar el disfrute equitativo de los derechos sociales en el ámbito educativo. Por lo tanto, el acontecer de la humanidad en todos sus ámbitos; cultura, identidad, realidades efectivas, conceptuales, imaginarias, virtuales, entre otras, se manifiesta entrelazado en diversos proyectos espaciales y temporales que permiten la flexibilidad de las potencialidades socio afectivas, cognitivas lingüísticas físicas y motoras del individuo en todas las facetas de su vida personal o colectiva las cuales se van configurando en historias propias y ajenas, cercanas o distantes, en constante interacción donde de algún modo todo es posible desde lo perceptible hasta lo imaginario en un mundo heterogéneo, diverso, múltiple y, fundamentalmente contradictorio, indeterminado, azaroso e incierto, problemático, dudoso, enigmático y misterioso, inconcluso e inconcluyente, además de simbólico, metafórico, poético o artístico. Considerando que todo esto son dimensiones reales de la condición humana, entonces, lo humano es ahora y desde siempre, un proyecto inacabado que tiende siempre a buscar una luz dentro de un largo camino que avanza y retrocede de acuerdo a los paradigmas que justifican su razón de ser.

Acceder, entonces, al paradigma de la complejidad es inmiscuirse con los avances científicos y tecnológicos que engloban al mundo en su totalidad, donde la complejidad desde una perspectiva semántica hace referencia a fenómenos $u$ objetos que se componen de elementos diversos, los cuales 
establecen relaciones recíprocas entre sí y configuran un todo. Es en esta apreciación de lo complejo que se busca profundizar en los rasgos constitutivos del mundo real producto del desarrollo que en las últimas décadas ha experimentado la ciencia física y la concepción del conocimiento científico surgido a raíz de la modernidad la cual le confiere a la ciencia, la misión de disipar la aparente complejidad de los fenómenos, a fin de develar el orden simple al que se supone obedecen los fenómenos que se suceden. Ahora bien, la ciencia contemporánea se encuentra en proceso de transición entre los ideales de conocimientos propios de la racionalidad clásica surgida a la luz de la modernidad, y una emergente racionalidad que desde diversas teorías científicas viene desplazando la simplificación desde la cual las disciplinas científicas creyeron desentrañar los enigmas del mundo. Hoy esta propuesta epistemológica es expresada bajo el término complejidad.

En este sentido Morín (2001), dice que "el pensamiento complejo está animado por una tensión permanente entre la aspiración a un saber no parcelado, no dividido, no reduccionista, y el reconocimiento de lo inacabado e incompleto de todo conocimiento". Es decir, el problema del sujeto no es un problema de subjetividad, sino que es la interrogación fundamental sobre sí mismo, sobre la realidad y la verdad.

Es aquí, donde la educación intercultural expresada, en términos de conocimiento y en la vía de obtención del saber, no es un aspecto más a considerar, sino un elemento fundamental del cual depende el valor y la confiabilidad del conocimiento, entendiendo que la cultura es una construcción permanente, es memoria y dimensión inherente a la persona humana en su sociedad; es particular y dinámica, comunitaria y universal, espiritual y material. Es también la visión y el orden del mundo y la vida, concebida a través de la sucesión de experiencias y descubrimientos, y de asociaciones que orientan el comportamiento de la persona en su familia y la sociedad. 
En tal sentido, la cultura es una fuente dinámica de cambio, creatividad y libertad que abre posibilidades de innovación y empoderamiento, al mismo tiempo que conocimiento y reconocimiento de la diversidad. De allí, que la interculturalidad se origina de la filosofía del pluralismo cultural, y va más allá del enfoque sociocultural y político del multiculturalismo. La interculturalidad es un diálogo entre iguales, en un contexto multilingüe, multiétnico y pluricultural. Al respecto Carlos Giménez Romero nos sintetiza que, "la interculturalidad es una relación de armonía entre las culturas; dicho de otra forma, una relación de intercambio positivo y convivencia social entre actores culturalmente diferenciados", por tanto, la interculturalidad es un camino para la construcción de la paz y la convivencia armónica, mutuamente enriquecedora entre los pueblos y comunidades: fortalece la unidad nacional por medio de la conducta fraternal entre sí de todos los habitantes. Los valores de todas las culturas que conforman la nación multicultural consolidan el bien común, la justicia y la equidad.

A partir de lo expuesto, se plantea como objetivo analizar los aspectos de la complejidad presentes en la educación intercultural.

\section{Bases Teóricas}

\subsection{La Complejidad}

Según Morín, E. (1990), al reflexionar sobre la complejidad señala que "los seres vivos permanecen en una constante auto organización en cada una de sus unidades sistémicas, donde además entran en interrelación con los elementos que los conforman".

Por lo tanto, la complejidad es una mezcla de orden y de desorden, ya que la sociedad está inmersa en esa constante auto organización e incertidumbre, donde se combinan diversas culturas, manifestaciones 0 formas de vida, hacen de ese conjunto de individuos una colectividad con cualidades múltiples y complejas. 
Dada la naturaleza de la complejidad, Doyle, C. (2010), reseña que a partir de finales del siglo XIX y principios del XX comienza a tomar forma el paradigma de la complejidad, uno de los momentos concretos en que esto ocurre es con la irrupción de lo complejo y del desorden en el universo físico, es decir, lo caótico y el azar son necesarios para lograr el orden. Los opuestos son complementarios y se articulan entre sí, se comienza a ver que la realidad es incompleta e imperfecta, el sistema no se puede reducir a la suma de las partes, es decir, que es más que ésta, lo reinante es la diversidad.

Con relación a lo expuesto, se destacan las características principales del paradigma de la complejidad apoyado en el estudio de Morín en el cual se enfocan en tres principios relacionados con el pensamiento complejo:

1. El principio dialógico: donde el orden y desorden son enemigos opuestos; pero en ciertos casos colaboran y producen la organización y la complejidad; la dualidad en el seno de la unidad.

2. El principio de la recursividad organizacional: un proceso recursivo en un sistema se da cuando los productos y efectos son, al mismo tiempo, causas y productores de aquello que los produce. Las interacciones de los individuos producen una sociedad, pero la sociedad produce a los individuos. Sin individuos no hay sociedad y sin sociedad no hay individuos.

3. El principio holo-gramático: la parte más pequeña de algo contiene la información de la totalidad de ese algo. Cada célula del cuerpo contiene la totalidad de la información genética, no se puede concebir el todo sin las partes y tampoco se pueden concebir las partes sin el todo.

De tal manera, que la complejidad muestra un mundo construido sobre una base caótica de variables articuladas que generan (o tratan de generar) sistemas de retroalimentación críticos. Todo depende desde donde se mire, por lo tanto, para poder ser capaz de conocer el mundo y tratar de tener una 
visión más abarcadora sobre él, se debe comprender que el contexto cultural que envuelve a las sociedades es muy profundo.

En el marco de la complejidad educacional, los estudiantes observan distinguen y explican cómo individualidades de interacciones con autoorganización, las características que determinan todo cambio en la sociedad subordinando y limitando dichos cambios a su propia conservación como organización. En los procesos educativos, los docentes son los encargados de explicar a sus educandos que la propiedad auto-poética (sistemas vivos, psíquicos y sociales, y serían la vida, la conciencia y la comunicación), que poseen como seres humanos trata de unidades organizadas como sistemas complejos que generan sus propios procesos de producción de componentes $\mathrm{y}$ relaciones entre ellos $\mathrm{y}$, a través de sus continuas interacciones $\mathrm{y}$ transformaciones se constituyen como unidades en un espacio físico determinado. En tal sentido, los estudiantes como complejos sistemas autopoéticos con capacidades auto-organizativas se reproducen a sí mismos, se levantan por sus propios medios, constituyéndose como personas diferentes a su entorno, crean desde sí su estructura y los elementos que lo componen tratando de mantener su identidad.

No obstante, la sociedad a través de la red de la información y la cultura digital que se vive en la actualidad ha profundizado la complejidad de la humanidad del conocimiento y la movilidad. En esta perspectiva, los estudiantes y docentes están obligados a asumir las nuevas coordenadas de ubicación interpersonal que ha trastocado los límites de los espacios convencionales con el surgimiento, de las redes sociales como Facebook, con la democratización simbólica, con la superación de software propietarios (Wiki, entre otros...), con la existencia de un ciberespacio eminentemente visual. Todo ello está impactando las relaciones individuales y de grupo, creando estructuras hipercomplejas sociales, generando con todo ello una nueva forma 
de integración social, un nuevo diseño del conocimiento. Es decir, se está viviendo una nueva forma de construcción social de la realidad.

Ante esta realidad, Morín (2001), plantea los siete saberes necesarios para la educación del futuro, los cuales resumiremos para su reflexión:

1.- Las cegueras del conocimiento: el error y la ilusión: La educación permanece ciega ante lo que es el conocimiento humano. En consecuencia, Morín considera que la primera e ineludible tarea de la educación para afrontar tal ceguera, ha de ser la de enseñar un conocimiento capaz de criticar el propio conocimiento en un escenario social de reflexibilidad crítica y sobre todo de convivencia ideológica.

2.- Los principios de un conocimiento permanente: Es necesario enseñar los métodos que permitan aprehender de las relaciones mutuas, influencias recíprocas entre las partes y el todo en un mundo complejo.

3.- Enseñar la condición humana: Porque es el objeto esencial de cualquier educación, ya que el ser humano es a la vez físico, biológico, psíquico, cultural e histórico. Para ello, el filósofo francés afirma que conocer el ser Humano es situarlo en el universo y, al mismo tiempo, separarlo de él: "la humanidad debe reconocerse en su humanidad común y, al mismo tiempo, reconocer la diversidad cultural implícita en todo lo humano.

4.- Enseñar la identidad terrenal, el desarrollo de un auténtico sentimiento de pertenencia a la tierra, considerada como imprescindible para el desarrollo de la conciencia antropológica, ecológica, cívica y espiritual.

5.- Una educación que enseñe a enfrentar las incertidumbres; en este saber, el autor, refiere las distintas sociedades que han prevalecido históricamente. Enfatiza que el futuro es incierto y el ser humano debe ser consciente de ello. No se ha educado para la incertidumbre, es importante fundamentar estrategias pedagógicas basadas en cada contexto, en cada realidad. 
6.- Enseñar la comprensión es otra de las preocupaciones de Morín, verificando y afirmando que la comprensión siempre está amenazada por los códigos éticos de los demás (sus costumbres, sus ritos, sus opciones políticas). De ahí, que los grandes enemigos de la comprensión sean el egoísmo, el etnocentrismo y el socio centrismo, por lo tanto, no se puede "etiquetar" las personas, ellas están más allá de la "etiqueta", por lo tanto, es importante reformar la mentalidad para que las relaciones humanas salgan del estado bárbaro de la incomprensión. Afirma que la comprensión alienta el establecimiento de sociedades democráticas, pues fuera de éstas no cabe la tolerancia ni la libertad para salir del círculo etnocéntrico. Concluye que la educación del futuro deberá asumir un compromiso sin requiebres con la democracia, porque sólo en la democracia abierta se puede realizar la comprensión a escala planetaria entre pueblos y culturas.

Reflexionando sobre los siete saberes anteriormente expuestos, es imprescindible no perder jamás la idea de transformación autónoma de aquel que es convocado al perfeccionamiento mediante el acto educativo. Sólo educando en el sentido auténtico del término se estará pensando en complejo y transmitiendo la complejidad en su total magnitud.

\subsection{Educación Intercultural.}

Yampara, S. (2001) expresa que:

Interculturalidad es, concertar dialogar -entre pueblos - entre los distintos modos de "saber hacer", los conocimientos, la sabiduría de las diversas civilizaciones en condiciones de equidad, respetando los derechos constituidos y la cultura. Es decir, compartir, complementar, intercambiar y reciprocar los saberes y valores de estos pueblos a través de procesos de tinkhus (encuentros) periódicos y continuos, respetando y forjando su identidad y dignidad (pág. 27) 
Bajo esta reflexión, se percibe la interculturalidad como el medio que orienta a forjar el respeto mutuo, poner en la balanza de la historia los valores éticos y ecológicamente compatibles y que históricamente, nos han dejado como herencia a través de una riqueza extraordinaria de elementos que aún persisten y que vale la pena mantener en el devenir de los tiempos.

Ahora bien, la interculturalidad en educación se considera como un modelo educativo en construcción, con demanda de una práctica pedagógica basada en el reconocimiento de la diversidad cultural de los pueblos, con participación, interacción y respeto, reconociendo y partiendo del contexto donde se origina.

Para Aguado (2005:52):

la interculturalidad como paradigma educativo ha venido desarrollándose casi exclusivamente en la última década y ha estado íntimamente relacionado con el desarrollo de la competencia lingüística, de la capacidad multilingüe, la lucha contra el racismo y la xenofobia y; el desarrollo de la educación intercultural, empezando por la búsqueda y construcción de un nuevo concepto de cultura.

Al respecto, Dietz y Mateos (2008:33), señalan que "la incapacidad manifiesta de las sociedades mayoritarias de hacer frente a heterogeneidad, impiden la consolidación de educación intercultural". Partiendo de estas consideraciones, la educación intercultural percibe ofrecer a todos los individuos las mismas oportunidades a través de disposiciones universales que se espera beneficien a los más necesitados.

Desde este punto de vista, la educación intercultural se comienza a utilizar en el discurso político y es por ello que la Organización Mundial de las Naciones Unidas (ONU), en 1985 declara el "Año Internacional de la Juventud" en ocasión de dirigir la atención hacia tres temas vinculados con la interculturalidad: la participación, el desarrollo y la paz. 
En virtud de este precepto, una década después, la Comisión Económica para América Latina y el Caribe (CEPAL), Ilamó la atención sobre la necesidad de incrementar las oportunidades de los jóvenes, sobre todo de aquellos más postergados de las zonas rurales y urbanas, pues ellos en medio de todas las limitaciones que se presentan, los jóvenes deben ser líderes y emprendedores, desde la escuela, que sean capaces de buscar alternativas de solución ante la diversidad de problemas que se presentan a lo largo de la vida, extendiéndose en el contexto del siglo XXI un nuevo precepto en la educación: el desarrollo de capacidades en pensamiento sistémico, de trabajo en equipo, de abstracción y de aprender a experimentar, lo cual prepara al joven para tener la facilidad de encontrar salidas adecuadas y pertinentes.

Atendiendo a estas consideraciones, la CEPAL plantea, que los jóvenes, deben estar en condiciones de generar medios para poder vivir aprovechando al máximo el potencial con que cuentan afianzando los procesos cognitivos para el mundo productivo, como alcance a la calidad de vida y progreso de las naciones. De allí, que la interculturalidad sea considerada como uno de los principios del contexto histórico en muchos países, porque promueve el desarrollo de las capacidades y actitudes de las personas en los procesos de construcción de la identidad acorde al reconocimiento y valoración de la diversidad cultural.

La educación intercultural en América Latina, por ejemplo, se ha implementado a través de la integración de los grupos autóctonos al sistema escolar, producto de la innovación educativa en el último cuarto de siglo XX donde se plantea una posible migración de los discursos interculturales entre países anglosajones y europeos continentales y, entre éstos y América Latina, lo cual ha sido motivo de reflexión por las diversas organizaciones para abordar la problemática de estas poblaciones. Desde este punto de vista, se vienen desarrollando diferentes programas de formación en interculturalidad en función de tres objetivos básicos: la enseñanza de la lengua castellana 
tanto en horario escolar como extraescolar, el mantenimiento de la lengua y la cultura de origen, el fomento de la convivencia democrática y el respeto a la diversidad. Además de ello, que aborde medidas en diferentes ámbitos (normativo, socio laboral, educativo, cultural, convivencia territorial y de participación social).

Al respecto, López (2001:75), refiere que las propuestas educativas para los grupos indígenas planteadas desde los gobiernos y desde los organismos de cooperación internacional se basaban en la integración a la sociedad criolla nacional desde la aculturación y asimilación lingüística. Posteriormente, fruto de tales demandas, surgió la "educación bilingüe de transición" que "...reconocía el uso diferenciado de los idiomas en conflicto y recurría al uso transitorio de los idiomas indígenas, por lo menos para facilitar la apropiación de un idioma europeo, así como del código escrito en éste". De esta manera nace, la educación bilingüe intercultural (o intercultural bilingüe) como una educación enraizada en la cultura de referencia de los educandos abierta a la incorporación de elementos y contenidos provenientes de otros horizontes culturales. Es una educación vehiculada en un idioma natal y un segundo idioma que propicia el desarrollo de la competencia comunicativa de los educandos en dos idiomas. La interculturalidad vista desde esta perspectiva, es todo un reto, es dar paso a una democracia práctica, no discursiva. Como señala Gimeno (2001:18) "al Estado no le corresponde decidir qué valores orientarán a la sociedad y a las culturas, sino garantizar las condiciones para que los grupos sociales y pueblos indios hagan efectivos sus proyectos".

\section{Metodología}

Metodológicamente fue una investigación de naturaleza analítica y documental por lo que se encuentra inserta en los estudios de desarrollo teórico. Éstos, según el Manual UPEL (2003:16), consisten en "la presentación 
de nuevas teorías, conceptualizaciones o modelos interpretativos originales del autor, a partir del análisis crítico de la información empírica y teorías existentes." De igual manera, para Arias (2006:27), la investigación documental es un proceso basado en "la búsqueda, recuperación, análisis, crítica e interpretación de datos secundarios, es decir, los obtenidos y registrados por otros investigadores en fuentes documentales: impresas, audiovisuales o electrónicas.

Por ende, para alcanzar el objetivo previsto se realizó una investigación analítica en la cual se consideró el proceso de análisis que propone Sánchez (2000), cuyo procedimiento residió en recorrer los siguientes pasos:

1.- Se definió el objetivo consistente en analizar los aspectos de la complejidad presentes en la educación intercultural.

2.- S eligió el tipo de análisis por elementos.

3.- Se definieron, describieron y analizaron: la Teoría de la Complejidad (Morin, 2008, 2001a, 2001b) y la Teoría de la Educación Intercultural (Aguado 2005).

4.- Por último, se describieron los resultados.

Cabe señalar que estos resultados se entienden como algo provisional que podrá complementarse, reestructurarse sobre la base de validaciones y discusiones posteriores.

\section{Resultados}

Del análisis de las teorías de la complejidad y de la educación intercultural, se encontró que la complejidad se encuentra en la educación intercultural tomando en cuenta la autonomía individual y la participación comunitaria. En el orden de presentar los aspectos de manera clara se elaboró unos criterios a partir de la teoría de la complejidad y sus elementos se contrastaron con la educación intercultural. A continuación, se describe cada 
criterio, definido desde la complejidad y su modo de presentarse en la educación intercultural.

Principio dialógico: de acuerdo con Doyle (2010) "permite mantener la dualidad en el seno de la unidad, asocia dos términos a la vez complementarios y antagónicos". En la educación intercultural lo dialógico está presente en la educación intercultural bilingüe, pues fomenta, tal como lo expresa Yampara (2001): "el concertar, el dialogar entre pueblos, entre sus distintos modos de saber y hacer, reconociendo sus conocimientos y sabiduría, respetando en condiciones de equidad los derechos constituidos y su cultura, cuya expresión fundamental es su lengua". Aguado (2005) reafirma al respecto que "la educación intercultural, empieza por la búsqueda y construcción de un nuevo concepto de cultura, es decir, la aceptación de la diversidad cultural".

Sobre el particular se tomaron en consideración las propuestas del pensamiento complejo presentado por Morín (1990) en dos principios que destacaremos a continuación:

Principio recursivo: todo lo que es producido regresa sobre aquello que lo ha producido en una especie de ciclo continuo, así mismo auto - constitutivo, auto - organizador y auto - productor; es decir, el individuo da origen a la sociedad al mismo tiempo que la sociedad produce al individuo. En ese orden de ideas, la educación intercultural es la responsable de mantener la diversidad cultural en el país, de tal forma que los individuos de las diversas culturas conserven sus valores culturales y los reproduzcan en/con las nuevas generaciones, sin estos individuos no habrá sociedad indígena y sin sociedad indígena no habrá individuos de las diversas culturas.

Principio hologramático: la complejidad no sería la respuesta, ella es un desafío cognoscitivo, no sólo impedirá toda teoría unificadora, sino que reconoce la unión de las partes, la contradicción y el reconocimiento de lo irreductible verificar la información, la educación intercultural es la conducta 
que rige el colectivo humano, existe una complementariedad compuesta de un todo.

Para Dietz y Mateos (2008:33), "la educación intercultural percibe ofrecer a todos los individuos las mismas oportunidades a través de disposiciones universales que se espera beneficien a los más necesitados".

Desde esta perspectiva la complejidad, es un fenómeno objeto de estudio para trasformar la realidad humana, la educación intercultural tiene como finalidad lograr desde la escuela actitudes de concertación entre poblaciones en un ambiente de convivencia en la colaboración y el respeto a los pueblos culturales.

La educación Intercultural vista desde la complejidad debe plantear entre sus objetivos reconocer individual y colectivamente que somos territorios con una gran diversidad cultural, enfrentándonos a problemas muchas veces parecidos y que si actuamos de manera intersolidarias podremos encontrar puntos de encuentro que nos permita solidarizarnos y tolerarnos en una sociedad que sea capaz de unir los conceptos que se rechazan entre sí y explicar lo visible complejo con lo invisible simple. Por ejemplo, para Morín existe una variedad de culturas con cualidades diversas de acuerdo a su espacio. Y Aguado (2005) nos advierte que "la educación intercultural se origina a partir del estudio de las relaciones existentes entre los indígenas y no indígenas es decir unirse a los pueblos indígenas y otras poblaciones".

La educación intercultural, parte también del Principio de Antopoiesis, que según Doyle (2010) es la comunicación y transformación de sistemas complejos, para organizar y mantener su identidad. Es decir, la educación intercultural es la capacidad auto organizativa y un sistema social.

En Venezuela actualmente las propuestas realizadas sobre la educación intercultural están orientadas de acuerdo al Currículo Bolivariano (2007) y se desarrolla por medio de cambios curriculares que progresivamente 
se adaptan en los procesos cognitivos y las innovaciones educativas propuestas en práctica la pedagogía de la educación.

En este apartado debemos tomar en cuenta a Morín (2001), quien recalca la importancia de desarrollar valores y actitudes que permitan fortalecer su dignidad, e identidad, a partir de la apropiación de los procesos históricos desde su realidad, valoración de los hechos vividos por los distintos hombres forjadores de la libertad en búsqueda de conocimientos. Para Aguado (2005), la Educación intercultural se convierte en un proceso social que emerge de la raíz de cada pueblo, orientado a desarrollar el potencial creativo de cada ser humano en la axiología de la diversidad cultural para alcanzar el pleno ejercicio de su personalidad y mantener la lengua, cultura basada en los procesos de transformación social involucrando los mismos.

\section{Conclusiones}

Es importante comprender que la complejidad aparece como la forma de captar la multidimensionalidad, las interacciones, las solidaridades, entre los innumerables procesos. Es una mezcla de orden y de desorden. Por tanto, la sociedad inmersa en esa constante auto organización e incertidumbre, donde se combinan diversas culturas, integrando la inseguridad en la esperanza situará los contenidos en los nuevos escenarios, comprendiendo así el universo, la tierra, la vida, lo humano, con cualidades múltiples y complejas.

El planteamiento sobre el desarrollo de un pensamiento complejo luce como una necesidad para transformar la realidad humana, como una especie de desafío a la hora de abordar y razonar todo lo que nos rodea. Por lo tanto, para poder ser capaz de conocer el mundo y tratar de tener una visión más abarcadora sobre él, se debe comprender que el contexto cultural que envuelve a las sociedades es muy profundo. 
La educación intercultural, surge como la necesidad de lograr desde la escuela actitudes transformadoras en un ambiente de convivencia en la colaboración, la tolerancia y el respeto ante la diversidad. Actitudes que nos lleven a propiciar nuevas formas de enseñar y de aprender, promoviendo formas críticas y creadoras de pensar, aumentando la autonomía del educando para la construcción de su proyecto de vida personal y comunitaria. Para que pueda tomar decisiones basadas en sus propios juicios y valores desde el ámbito del propio grupo cultural y del grupo cultural dominante.

La educación intercultural plantea objetivos acordes a estos tiempos de la globalización, desde el entendido de que tenemos que reconocer que somos un país con una gran diversidad cultural, y que para construir una Identidad cultural sólida, tenemos que tener siempre presentes las raíces de identidad que nos unen como venezolanos, haciendo conciencia de nuestros símbolos: Étnicos, lengua, tradiciones, costumbres religión e historia, fortaleciendo cada día las fuerzas de identidad plural en la de unidad nacional.

\section{Referencias}

Aguado (2005). Educación intercultural y aprendizaje cooperativo. Madrid: Pirámide

Arias, F. (2006). El proyecto de investigación. Introducción a la metodología científica. 5ta edición. Caracas, Venezuela: Episteme.

Dietz y Mateos (2008). Cuando inmigrantes y autóctonos comparten estereotipos: niños, escuela e imágenes sobre la inmigración marroquí en España". Revista Anales de Historia Contemporánea, Vol. 15, pp. 167-177.

Doyle (2010). Relaciones Interpersonales Interculturales en Educación Secundaria Obligatoria. X Congreso Nacional de Modelos de Investigación Educativa. Investigación y sociedad en la sociedad del conocimiento. La Coruña: AIDIPE. 
Morín, Edgar (2001). La cabeza bien puesta. Nueva Visión. Buenos Aires.

Recuperado de: http://www.edgarmorin.org/descarga-la-cabeza-bienpuesta.html

Morín, E. (1999). Los siete saberes necesarios para la educación del futuro. UNESCO. Editorial Santillana.

Freire, P. (1980). Pedagogía del oprimido. Siglo XXI Editores. México.

Sánchez, M. (2000). Desarrollo de habilidades del pensamiento. 10ma. reimpresión. México: Trillas.

Universidad Pedagógica Experimental Libertador, Vicerrectorado de Investigación y Postgrado. (2011). Manual de trabajos de grado, de especialización y maestría y tesis doctorales. 4ta. edición, 11va. reimpresión. Caracas.

Wittgenstein, L. (2003). Tractatus logico-philosophicus. Libro de bolsillo. Alianza Editorial. Madrid.

Yampara (2001). Estrategias didácticas y organizativas ante la diversidad: dilemas del profesorado. Barcelona: Octaedro. 


\section{María del Carmen Pérez Paredes \\ e-mail: miduam@hotmail.com}

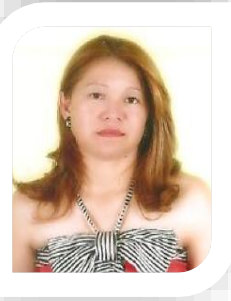

Nacida en Timotes estado Mérida, cursa estudios de Doctorado en la Universidad Rafael María Baralt, Magister Scientiarum en Administración de la Educación Básica, en la Universidad Rafael María Baralt, estado Zulia (2012), Licenciada en Educación en la Universidad Alonso de Ojeda, Estado Zulia, (2008), Abogada de la República, egresada de la Universidad Bolivariana de Venezuela Misión Sucre (2011).

Actualmente me desempeño como Coordinadora Pedagógica adscrita al Núcleo Rural 196 del Municipio Miranda del estado Mérida, desde el año (2016). Me desempeñe durante 12 años como secretaria en la Prefectura Civil adscrita a la gobernación del estado Mérida, experiencia como docente de aula en la escuela rural Bolivariana El Paramito desde el año 2007 hasta el año 2015, actualmente pertenezco al escritorio Jurídico Dr. Leonardo Rivas \& Asociados, Timotes Mérida. Labore desde el año 2013 al 2014 como profesora de FEVU en la UNEFA, estado Trujillo. Participante como jurado evaluador de tesis de grado en la UNES durante el año (2013).

Ponente y participante con temáticas relacionadas diferentes líneas de investigación, desde el año 2013, hoy día se traza como meta la investigación etnográfica en una Comunidad Indígena, así como dictar talleres sobre leyes CRBV, LOPNNA, Consejos Comunales, Ley de la Mujer.

El contenido de este manuscrito se difunde bajo una Licencia de Creative Commons ReconocimientoNoComercial-Compartirlgual 4.0 Internacional 